\title{
DE LA DATE DES GLOSES DE SILOS
}

\author{
Francis BezLer \\ Strasbourg
}

En 1926 R. Menéndez Pidal publie ses Orígenes del español, où il donne la première édition complète des glosas silenses ${ }^{1}$. Se fondant sur les traits paléographiques du manuscrit contenant ces gloses ${ }^{2}$, il date le tout, manuscrit et gloses, de la seconde moitié du $\mathrm{x}^{e}$ siècle $^{3}$. Et c'est cette date qui est encore généralement retenue par ceux qui abordent le sujet ${ }^{4}$.

Avant Menéndez Pidal, le manuscrit passait pour dater du $\mathrm{xI}^{e}$ siècle ${ }^{5}$. Dom Férotin le situait même à la fin du $\mathrm{xI}^{\mathrm{e}}$ siècle $^{6}$, tandis que Dom Morin n'hésitait pas à le dater du début du XII $\mathrm{I}^{*}$ siècle?

L'argumentation de ce dernier reprend et développe celle qui est ébauchée dans la notice du Cataloguc of Additions. Ainsi, Dom Morin signale que l'on peut nettement distinguer deux parties dans le manuscrit: une première partie contenant les lectures de toute l'année liturgique, allant jusqu'au folio

1 Ramón Menéndez Pidal, Origenes del español. Estado lingüístico de la Península Ibérica hasta el siglo XI, Madrid, 1926, 9. éd., 1980, pp. 9-24.

2 Manuscrit conservé à la British Library, cf. Catalogue of Additions to the manuscripts in the British Museum in the years 1876-1881, Londres, 1882, p. 121, Add. 30.853.

3 Origenes, p. 9.

- Ainsi: Antonio Quilis, Historia de la lengua española, Univ. Nac. de Educación a Distancia, Madrid, fasc. 2, 1976, p. 6; C. Tagliavini et A. Niculescu, Originile limbilor neolatine, Bucarest, 1977, p. 414; Norberto Núñez, «Silos, mil años de lengua castellana», Castilla. El libro del milenio de la lengua (Federico Pérez, dir.), Burgos, 1979, pp. 93-96; Joan Corominas et J. A. Pascual, DECH, 1, 1980, p. XLIXb; Rafael Lapesa, Historia de la lengua española, Madrid, 1942, $9^{\circ}$ éd. 1985, p. 162 («... siglo x, o comienzos del xI.»).

s Catalogue of Additions, p. 121; Josef Priebsch, "Altspanische Glossen», $\mathrm{ZrPh}, 19$, 1985, p. 1; Charles Upson Clark, Collectanea Hispanica, Paris, 1920, p. 38, n. 566; Zacarfas García Villoslada, Paleografía española, Madrid, 1923 (reproduction en offset dans Obras Completas, Barcelone, El Albir, 1974), t. I, p. 104, n. 70.

6 Marius Ferotin, Le Liber Mozarabicus Sacramentorum et les manuscrits morazabes, Paris, Firmin Didot, 1912, col. 882.

7 Germain Morin, Liber Comicus, dans Anecdota Maredsolana, t. I, 1893, p. 406. 
231 où le copiste, arrivé au terme de son travail, écrivit les mots: FINI DEO GRATIAS AMEN; une deuxième partie contenant le reste des textes (dont le pénitentiel avec les gloses), provenant de la même collection originelle que les homélies précédentes, ou d'autres sources. Or, c'est entre ces deux parties qu'est insérée une lettre apocryphe qu'un certain Pierre, évêque de Nimes, assure avoir reçue du Seigneur lui-même. De là Dom Morin déduit, puisqu'il ne peut s'agir que de Pierre Ermengaud, qui a dirigé l'Eglise de Nimes dans les années 1080-1090, que le manuscrit doit dater du début du $\mathrm{xII}^{\mathrm{e}}$ siècle.

Menéndez Pidal n'a jamais fait la moindre allusion à cette argumentation. Était-ce, comme on a pu l'écrire ${ }^{8}$, qu'à ses yeux elle n'en valait pas la peine et qu'elle méritait d'être passée sous silence? N'était-ce pas traiter un peu à la légère un savant érudit comme Dom Morin et un fin connaisseur des manuscrits wisigothiques comme Dom Férotin?

Par ailleurs, la date très précise de 1052 fut attribuée au pénitentiel de Silos par le numismate Pío Beltrán Villagrasa dans un article (guère remarqué des philologues) publié en $1952^{9}$. L'auteur y étudie les données monétaires figurant dans ledit pénitentiel. Mais il utilise l'édition qu'en a donnée au XVIII ${ }^{e}$ siècle le P. Francisco de Berganza ${ }^{10}$, et comme celui-ci affirme que les textes publiés par lui dans la Sección Tercera de son ouvrage (entre autres, le pénitentiel) sont tirés d'un manuscrit daté de $1052^{11}$, Pío Beltrán en conclut à l'existence d'un système monétaire propre à la Castille dès $1052^{12}$.

Vérification faite, on constate que, si Francisco de Berganza utilise bien un manuscrit daté de 1052 pour une bonne partie des textes publiés dans sa Sección Tercera, il tire les Cánones Penitenciales utilisés par Pío Beltrán (en fait, le pénitentiel de Silos) d'un autre manuscrit, sans le dire. Ce manuscrit est évidemment le codex Add. 30.853. Donc, la preuve alléguée par Pío Beltrán pour étayer sa thèse s'effondre.

Enfin, bien plus récemment, Manuel C. Díaz y Diaz est arrivé à la conclusion qu'il faut distinguer deux parties dans le manuscrit Add. 30.853: les 308 premiers folios, comprenant un homiliaire "escrito con gran atención, en

8 Georges Straka, «Pour une révision de la date des gloses de Silos?», Hommage d Bernard Pottier, Paris, Klincksieck, 1987, t. II, p. 753.

- Pío Beltrán Villagrasa, «Dinero de vellón de Fernando I el Magno en la colección Los Arcos», 1952, repris dans Obra Completa. II. Numismática de la Edad Media $y$ de los Reyes Católicos, Saragosse, 1972, pp. 585-605.

10 Francisco de Berganza, Antigüedades de España, t. II, Madrid, 1721, p. 666 sq.

11 Ibid., p. 624.

12 Affirmation et tableau de ce prétendu système monétaire catillan repris par Antonio Ubieto Arteta dans: A. Ubieto-J. Reglá-J. M. Jover-C. Seco, Introducción a la bistoria de España, Barcelone, Teide, 1963, 4e éd. 1967, p. 138, ouvrage de grande diffusion. 
letra clara $\mathrm{y}$ elegante de finales del siglo $\mathrm{x}$, o primeros decenios del $\mathrm{xI}$ " ${ }^{13}$; et les folios 309-324, où figure le fameux pénitentiel avec glosses, dont l'écriture est "netamente silense, de la segunda mitad del siglo xI" ${ }^{14}$.

Si l'écriture du pénitentiel de Silos est de la deuxième moitié du $\mathrm{xI}^{\circ}$ siècle, cela ne signifie pas encore que les gloses ne datent pas de la deuxième moitié du $\mathrm{x}^{e}$ siècle, comme le voulait Menéndez Pidal. On sait en effet que le manuscrit est une copie, et les gloses probablement aussi. L'original peut en être beaucoup plus ancien. Heureusement, le pénitentiel présente certaines particularités, comme ses listes de commutations, qui peuvent nous permettre de préciser la date de son élaboration.

Disons d'abord en peu de mots, pour la bonne intelligence de l'exposé, ce qu'il faut entendre par pénitentiel et par liste de commutation.

Un pénitentiel, ou liber paenitentialis, est un écrit de dimension variable contenant des listes de péchés, affectés chacun d'une taxe ou tarif d'expiation. Ce tarif consiste presque toujours en un nombre déterminé de jours, de mois ou d'années de jeûne, ou d'autres oeuvres de piété ou de charité. Parce que chaque péché de ces listes est affecté d'un tarif expiatoire précis, la pratique pénitentielle que ces livrets documentent est appelée pénitence tarifée.

Issue des chrétientés celtiques, elle se répandit sur le continent à partir du $\mathrm{vI}^{\bullet}$ siècle (saint Colomban 540-616) et y entraina une prolifération de libri paenitentiales dont la vague atteignit aussi l'Espagne.

Les listes de commutations étaient indispensables au bon fonctionnement du système de la pénitence tarifée. En effet, comme les tarifs des différents péchés s'additionnaient généralement, un pécheur moyen pouvait, après confession, se voir imposer une expiation (presque toujours un jeûne) s'étendant sur de longues années. Seules des âmes d'élite pouvaient se soumettre à un pareil régime, et le système risquait de ne pas pouvoir être mis en pratique pour le commun des fidèles. C'est pourquoi il comportait un correctif: les commutations, ou rédemptions, qui permettaient au pénitent de "racheter" son jeûne pénitentiel de diverses façons (par des prières, des génuflexions, des récitations de psaumes, des flagellations, des amendes en numéraire, etc. ${ }^{15}$.

L'auteur du pénitentiel de Silos a, lui aussi, élaboré des listes de commutations, en appendice à son livret. Il y envisage d'abord le cas des clercs, puis

13 Manuel Cecilio Díaz y Diaz, Códices visigóticos en la monarquía leonesa, León, Centro de Estudios e Investigaciones San Isidoro, 1983, p. 317, n. 26.

14 Ibid., p. 405, n. ${ }^{\circ}$ 126. Si Georges Straka n'avait pas ignoré cet important ouvrage, il se serait abstenu d'écrire son malheureux article ci-dessus mentionné, supra note 8.

15 Pour une plus ample information sur ces questions, voir: Cyrille Vogel, Les Libri Paenitentiales, Typologie des sources du Moyen Age occidental, fasc. 27, Turnhout, Brepols, 1978; Raymund Kottje, «Bussbücher», Lexikon des Mittelalters, t. II, 1982, col. 1118 sq. 
celui des laics. Ces derniers sont répartis en différentes catégories, chaque pécheur devant verser, pour racheter son jeùne, une certaine somme d'argent, selon la catégorie à laquelle il appartient. Cela nous donne la liste suivante (retranscrite d'après une photocopie):

$\mathrm{Si}$ imperator est, solidum unum exsolbat. Princeps, V argenteos. Comes, IIIIor. Amirates, III. Equestres, II. Operator rurium qualiumquumque, I argentum. Mercenarius, semis argentos. Pauper, obolum quod vulgo quarta dicitur. Pauperrimus, unam seliquam, id est harrobam.

Cette liste est ensuite répétée avec les tarifs pour le rachat d'un jeûne de quarante jours.

Comme on voit, le clerc espagnol calcule la commutation en numéraire des laïcs d'après leur rang dans la société. Il nous offre ainsi la hiérarchie sociale de son époque (du moins telle qu'il la conçoit). Au sommet de cette échelle est placé l'Imperator. Or, ce titre d'Imperator fut utilisé en Espagne, pendant un temps, pour désigner certains souverains.

Ce furent en effet les rois de Léon qui adoptèrent le titre impérial à partir du $\mathrm{x}^{8}$ siècle $^{16}$. Ce titre tomba rapidement en désuétude et disparut dans la première moitié du $\mathrm{xII}^{\mathrm{e}}$ siècle. Si dès le début il impliqua un sentiment de supériorité de la part des rois de Léon sur les autres royaumes chrétiens de la Péninsule, ce n'est qu'au cours du $\mathrm{xI}^{\mathrm{e}}$ siècle que le terme Imperator acquit clairement le sens de Roi supérieur aux autres rois. L'idée impériale espagnole atteignit son apogée sous les règnes d'Alphonse VI, Imperator totius Hispaniae (1072-1109), et d'Alphonse VII (1126-1157), couronné empereur d'Espagne en la ville de Léon en 1135 .

Le terme Princeps qui suit immédiatement celui d'Imperator dans la liste de commutations, servait communément à désigner, à la même époque, le roi, et lui seul ${ }^{17}$. En effet, alors que le titre de Rex était porté non seulement par le monarque (et sa femme, la Regina), mais aussi par son fils ainé, et même parfois par plusieurs de ses enfants simultanément (en particulier lorsqu'ils étaient chargés de l'administration d'une partie, fût-elle petite, du territoire), le titre de Princeps était exclusivement réservé à celui qui exerçait effectivement le pouvoir sur l'ensemble du royaume.

Dans le royaume de Léon, l'Imperator et le Princeps étaient évidemment une seule et même personne. Donc, l'horizon de l'auteur de ces commutations

16 Luis García de Valdeavellano, Curso de bistoria de las instituciones españolas. De los origenes al final de la Edad Media, $5^{\circ}$ éd., Madrid, Biblioteca de la Revista de Occidente, 1977, p. 228 sq.

17 José Antonio Maravall, Estudios de bistoria del pensamiento español, Serie Primera: Edad Media, 2e éd., Madrid, Ediciones Cultura Hispánica, 1973, p. 33 sq.; idem, El Concepto de España en la Edad Media, $2^{-}$éd., Madrid, Centro de Estudios Constitucionales, 1964, p. 341 sq. 
ne se limitait pas aux frontières de ce seul royaume, car la distinction entre l'un et l'autre titre eût été superflue. Il envisageait manifestement, et logiquement (puisqu'il visait le pécheur, tous les pécheurs) la société chrétienne espagnole dans son ensemble. Une société caractérisée par une hiérarchie précise, d'après laquelle le Princeps, c'est-à-dire n'importe lequel des rois espagnols d'alors, venait après l'Imperator.

Il se dégage donc de ces commutations, très clairement nous semble-t-il, une volonté d'embrasser la société espagnole dans sa totalité, dans son unité fondamentale de chrétienté, de l'unifier dans une même discipline ecclésiastique, la discipline pénitentielle, qui vise en même temps à la réformer moralement, et de l'organiser en une hiérarchie au sommet de laquelle est placé l'Imperator.

Volonté de réforme et exaltation de l'Imperator audessus des autres rois sont deux caractéristiques du règne de Ferdinand $\mathrm{I}^{\mathrm{er}}$, Roi de Castille et Empereur hispanique (1037-1065). C'est en effet avec Ferdinand I ${ }^{\text {er }}$ que l'idée impériale s'affirme dans sa plénitude, le titre étant officiellement reconnu par les frères de l'empereur, les souverains de Navarre et d'Aragon, qui ne s'attribuèrent que le titre de roi $^{18}$. C'est aussi Ferdinan $\mathrm{I}^{\mathrm{er}}$ qui, dans son désir de pacifier ses royaumes et d'y remettre de l'ordre, prit l'initiative de réunir le seul concile, autant que l'on sache ${ }^{19}$, qui se soit tenu en Espagne depuis l'effondrement du royaume wisigothique: le concile de Coyanza de 1055, qui devait s'occuper, entre autres, de la remise en état de l'Eglise et de la réforme morale du clergé ${ }^{20}$.

Donc, le clerc espagnol auteur de la liste de commutations de notre pénitentiel a plutôt vécu vers le milieu du $\mathrm{xI}^{\mathrm{e}}$ siècle, et non au $\mathrm{x}^{\mathrm{e}}$.

Tournons-nous maintenant vers les unités monétaires utilisées par notre clerc pour fixer les tarifs de ses commutations. Pío Beltrán les a soigneusement étudiées. En confrontant les tarifs pour un jour de jeûne avec les tarifs pour quarante jours il a calculé le rapport entre les différentes unités, et dressé le tableau de ce qu'il croyait être un système monétaire castillan ${ }^{21}$. Le terme harrobam utilisé par l'auteur du pénitentiel aurait cependant dû le mettre sur la bonne voie. Il s'agit en effet là de la retranscription en caractères latins du mot кHarrüba, désignant une unité du système monétaire

18 Valdeavellano, Curso, op. cit., p. 230; idem, Historia de España. De los orígenes a la baja Edad Media, Madrid, Alianza, 1980, vol. 2, pp. 273-274.

${ }_{19}$ Jean Gaudemet, Les sources du droit de l'Eglise en Occident du II $I^{e}$ au VII siècle, Paris, éd. du Cerf, 1985, p. 160 n. 108: «Le premier concile de la Péninsule, après le VIII ${ }^{e}$ siècle, se tiendra en 1055 ».

${ }_{20}$ Alfonso García Gallo, «El concilio de Coyanza. Contribución al estudio del derecho canónico español en la alta Edad Media», Anuario de bistoria del derecho español, Madrid, Instituto Nacional de Estudios Jurídicos, t. XX, 1950, pp. 275-633.

21 Cf. supra, note 9. 
arabe. Les solidos et les argentos de notre pénitentiel ne sont rien d'autre que des dinars (or) et des dirhems (argent) hispano-musulmans: ceux-ci avaient en effet cours dans l'Espagne chrétienne, où on les affublait d'un nom latin, comme le savent bien les spécialistes de l'histoire monétaire ${ }^{22}$.

Donc, le système monétaire connu et pratiqué par l'auteur du pénitentiel de Silos était en réalité le suivant:

\begin{tabular}{|c|c|c|c|c|}
\hline & sólido/dinar & argento/dirhem & quarta & harroba \\
\hline sólido/dinar ............ & .... 1 & 8 & 32 & 96 \\
\hline argento/dirhem $\ldots \ldots \ldots \ldots$ & $\ldots \ldots \ldots \ldots$ & $\ldots \ldots \quad 1$ & 4 & 12 \\
\hline quarta $\ldots \ldots \ldots \ldots \ldots$ & $\ldots \ldots \ldots \ldots$ & $\ldots \ldots \ldots \ldots$. & $\ldots 1$ & 3 \\
\hline harroba $\ldots \ldots \ldots c c c c$ & $\cdots \cdots \cdots$ & $\begin{array}{llllll} & \cdots & \ldots & \ldots & \ldots & \cdots\end{array}$ & $\ldots \ldots$ & 1 \\
\hline
\end{tabular}

Comparons maintenant ce tableau avec ce que l'on sait du système monétaire de l'Espagne islamique à son apogée, c'est-à-dire pendant le Califat de Cordoue (929-1031). Sans entrer dans le détail du sujet, très complexe ${ }^{23}$, retenons que les principales unités monétaires (pièces réelles ou monnaie de compte) de ce système étaient le dinar, le dirhem-kaïl (unité d'argent ayant le poids légal d'Orient), qui apparait dans les documents chrétiens sous le nom d'argenteos grossos; le dirhem-dokhl (unité d'argent propre à al-Andalus), qu'en pays chrétien on appelait simplement argento; la ruba, mot signifiant: le quart; et le kiñăt ou кнаrrūba. On voit donc que les termes dinar, dirhem-dokhl, ruba et kîuāt coïncident exactement avec les dénominations monétaires de notre pénitentiel, à savoir: solidus, argentum, quarta et harro$b a$. Soulignons cependant une différence importante: la ruba est un quart de dinar, alors que notre quarta est un quart d'argentum, donc de dirhem.

Autre différence notable: le rapport du dinar au dirhem. A l'époque du Califat de Cordoue un dinar valait dix-sept dirhems d'al-Andalus (ou argentos) ${ }^{24}$. Or, d'après le pénitentiel de Silos, un dinar ne vaut que huit dirhems.

La crise du Califat, sa désintégration en une multitude de taïfas, et la politique d'extorsion systématique de fonds (parias) auxquels les royaumes chrétiens du nord soumirent les roitelets hispano-musulmans, ne manquèrent pas de retentir sur la situation monétaire d'al-Andalus. Les rois de Taĩfas

22 Sur ces questions voir: Jean Gautier Dalche, «L'histoire monétaire de l'Espagne septentrionale du IXe au XII ${ }^{\mathrm{e}}$ siècle», Anuario de Estudios Medievales, 6, Barcelone, 1969, pp. 43-95.

23 Pío Beltrán Villagrasa, «El sistema monetario del Califato de Córdoba», Ligartzas, I, Valencia, 1968 , pp. 7-77.

24 Ibid. 
ne payèrent évidemment pas de gaieté de coeur les parias. Autant par manque de ressources que pour ménager leurs richesses, ils émirent de plus en plus souvent des pièces (d'argent surtout) de mauvais aloi. L'argent monta, l'or chuta, et les rapports entre le dinar et le dirhem furent bouleversés ${ }^{25}$.

Les textes qui nous fournissent des références monétaires sur cette période sont en général difficiles à interpréter clairement ${ }^{26}$. Cependant, la lecture de certains d'entre eux permet d'affirmer qu'il existait des dinars dont la valeur n'était pas la même selon qu'ils provenaient du royaume de Tolède ou du royaume de Saragosse, ou d'une autre Taifa, et nous fournit d'autre part quelques points de repère. Ainsi, en 1073 un dinar, appelé mancusus auri optimi, valait sept dirhems à Saragosse ${ }^{27}$. En 1083, mais cette fois à Tolède, deux ans avant l'occupation de la ville par Alphonse VI, le dinar, appelé cette fois mizcal, valait dix dirhems ${ }^{28}$. Le dinar/solidus à huit dirhems de nos tarifs pénitentiels s'intègre aisément à ce paysage monétaire. On peut même essayer de fixer, avec une précision relative, la date à laquelle ces tarifs durent être composés.

Le royaume de Castille-León, où notre pénitentiel fut élaboré, ne commença à percevoir des parias que dans les dernières années du règne de Ferdinand $\mathrm{I}^{\mathrm{er} 29}$. Tout le système des parias chancela lorsque les Almoravides,

25 Cf. Pío Beltrán Villagrasa, «El sistema monetario...», art. cit., p. 66: «Con el descenso de la ley de la moneda de plata durante las taifas disminuyó el valor del oro (por aumentar el valor de la plata) y resultan otros valores del dinar y del sólido aúreo, en 'argenzos'».

26 Cf. José María Lacarra, «Aspectos económicos de la sumisión de los reinos de Taifas (1010-1102)», Homenaje a Jaime Vicéns Vives, vol. I, Barcelona, 1965, pp. 267. 268: «No es posible hacer un cálculo de las cantidades percibidas por los príncipes cristianos en concepto de parias, ni tampoco del valor de los metales preciosos puestos en circulación en territorio cristiano como resultado del botín de guerra o como fruto de la actividad comercial. Los datos que nos facilitan las crónicas y documentos carecen, con frecuencia, de la precisión debida (...). Resulta también difícil calcular la equivalencia de monedas, dada la variedad de nombres que les aplican los textos, muchos de los cuales no nos han llegado en versiones contemporáneas (...)».

27 «Convenit Almuctadir ville (i. e. al-Muqtadir bi-llah) per singulos annos dare regi domino Sancio duodecim milia mancusos auri obtimi, ita ut si regi placuerit accipere aurum accipiat, si enim plus sibi placuerit accipere aurum pro unoquoque mancuso auri accipiat rex VII solidos argenti de moneta de Cesaraugusta», cité par: José María Lacarra, «Dos tratados de paz y alianza entre Sancho el de Peñalén y Moctádir de Zaragoza (1069 y 1073)", Homenaje a Jobannes Vincke para el 11 de mayo de 1962, vol. I, Madrid, C.S.I.C., Goerres Gesellschaft zur Pflege der Wissenschaft, 1963, p. 133.

${ }_{28}$ Cf. Ángel González Palencia, Los mozárabes de Toledo en los siglos XII y XIII, t. I, Madrid, Instituto de Valencia de Don Juan, 1926, p. 1: «Venta de la mitad de una viña $(\ldots)$ otorgada por Chamila, hija de Fárach, esposa de Belinsí el Albañil, a favor de Rabí Binshac ben Nehemías el judío, por precio de 300 mizcales (c-à-d. des dinars, mithkhāl étant l'unité pondérale) de los que ahora corren por Toledo, de a 10 dirhemes el mizcal, según la ley de los musulmanes (...)m.

${ }^{20}$ Cf. José María Lacarra, «Aspectos económicos ...», art. cit., p. 265: «Fue Fernando I, en los últimos años de su vida, quien, en campañas dirigidas por tierras de 
après l'occupation de Tolède par les hispano-chrétiens (1085) et la confiscation de la grande mosquée de la ville, franchirent le clétroit de Gibraltar pour venir à la rescousse de l'Islan d'Espagne, ranimèrent la flamme du Djihäd, et taillèrent en pièce l'armée du puissant roi de Castille-Léon, Alphonse VI, à la bataille de Zalaca, ou Sagrajas (près de Badajoz), en 1086.

Notre dinar/solidus à huit dirhems nous semble donc plutôt à rapprocher du dinar/mancusus à sept dirhems de Saragosse de l'an 1073 que du dinar/ mithkhãl à dix dirhems de la Tolède des années 1080. Si l'on considère qu'au cours du $\mathrm{xI}^{\mathrm{e}}$ siècle la monnaie n'a cessé de se dévaluer ${ }^{30}$, le dinar à huit dirhems devrait être antérieur au dinar à sept dirhems. C'est pourquoi on ne se trompera sans doute pas de beaucoup en datant nos tarifs de commutations des années 1060-1070.

Par ailleurs, certains canons de notre pénitentiel indiquent que la liturgie mozarabe est encore en vigueur ${ }^{31}$. Or, celle-ci fut officiellement remplacée, dans le royaume de Castille-Léon, par le rite romain sur décision d'Alphonse VI en l'an 1080. D'autre part, en 1055 fut réuni, sur ordre de Ferdinand $\mathrm{I}^{\mathrm{e}}$, le concile de Coyanza, aboutissement d'un mouvement réformateur à l'intérieur de l'Eglise d'Espagne, et surtout expression d'une volonté officielle de restauration institutionnelle et morale. Or notre pénitentiel, par son ampleur, par le soin avec lequel il fut composé, et par l'esprit dont il est empreint, nous semble devoir être considéré (quoiqu'il n'y ait pas de coincidence littérale avec les textes de Coyanza) comme une oeuvre élaborée dans le sillage de ce concile.

Pour toutes ces raisons, nous estimons que les tarifs de commutations du pénitentiel de Silos datent des années 1060-1065. Il est donc impossible que les glosses dont ce document est pourvu soient antérieures à cette date. Il est, au contraire, très probable que les glosas silenses soient d'un siècle plus jeunes que ne le prétenclait $R$. Menéndez Pidal.

Toledo, de Zaragoza y de Sevilla, logró que sus reyes le ofrecieran parias, que él distribuyó entre sus hijos, juntamente con sus reinos. Según la Crónica Compostelana, a Sancho dejó Castilla con los tributos anuales que le daba el rey de Zaragoza; a Alfonso, el reino de León, con lo que pagaba el reino de Toledo, y a García el reino de Galicia con lo que pagaban Sevilla y Badajoz (...)m.

${ }_{30}$ Cf. idem, ibid., p. 268: «(...) no hay que olvidar la devaluación que experimenta la moneda a lo largo del siglo XI (...)m.

31 «Mulier menstruosa in die sancte pasche resurrectionis tantum carnem benedicti agni edat»; «Si autem mortuus erit, nulla illi in oblatione commemoratio fiat neque cum psalmis aut sale ad sepulturam cadabera eorum deducantur (...)»; «Si virgo virgini coniuncta est sine velaminum benedictione (...)»; «Nullus gradum ecclesiasticum percipiat qui totum psalterium vel babtisterium aut ordinem salsparsionum vel sepulturarum perfecte structus non fuerit agendi officio». 